\title{
Spatial-Temporal Evolution and Spillover Effect of Industrial Eco-Efficiency in Huaihe River Economic Belt
}

\author{
Yanna Zhu ${ }^{1,2 *}$, Gang $\mathrm{He}^{1,2 * *}$, Guisheng Zhang ${ }^{1}$ \\ ${ }^{1}$ State Key Laboratory of Mining Response and Disaster Prevention and Control in Deep Coal Mines, \\ Huainan, Anhui, 232001, China \\ ${ }^{2}$ College of Economy and Management, Anhui University of Science and Technology, \\ Huainan, Anhui, 232001, China
}

Received: 11 June 2021

Accepted: 10 September 2021

\begin{abstract}
This paper takes Anhui section of the Huaihe River Economic Belt as the research object, and comprehensively uses SBM-Undesirable and DEA-Malmquist models to measure the industrial eco-efficiency from 2009 to 2018. With the help of ArcGIS and GeoDA software, the spatial characteristics of industrial eco-efficiency are analyzed. Based on Tobit panel regression model, the paper makes an empirical analysis on the influencing factors of industrial eco-efficiency, and discusses the mechanism of industrial eco-efficiency pattern evolution. The results indicate that: (1) On the whole, the level of industrial eco-efficiency from 2009 to 2018 is relatively low, and the difference of industrial eco-efficiency among eight cities is obvious. Spatially, the eco-efficiency index shows an increasing spatial pattern from north to south. (2) Most of the eight cities in Anhui section are in the input-output development mode of high benefit, medium consumption, low pollution and low benefit, low consumption and low pollution. (3) Economic development level and research innovation intensity have a positive promoting effect on industrial eco-efficiency. Urbanization level and the proportion of environmental protection investment have a negative inhibiting effect on the improvement of industrial eco-efficiency. The industrial structure and the degree of openness have no significant effect on industrial eco-efficiency.
\end{abstract}

Keywords: industrial eco-efficiency, SBM-Undesirable model, DEA Malmquist model, spatial-temporal pattern, influence factor 


\section{Introduction}

Industry is the main industry of economic development, which provides a lot of material basis for society and plays an important role in supporting human life and production. However, the development of industry is accompanied by the massive exploitation and consumption of fossil energy and the wanton emission of pollutants. The increasing rigid constraints of resources and environment are strengthened, and the contradiction between industrial development and ecological environment is becoming increasingly prominent. In November 2018, the Huaihe River Economic Belt Development Plan pointed out that it was necessary to develop the Huaihe River Basin into a green development belt, where here the sky was blue, the land was green, the water was clear, and harmonious coexistence of humans and nature. In recent years, the problems such as "high input, high consumption and high emission" still exist widely in this region. The economic development has constrained the sustainable development of this region at the cost of excessive resources and environment. How to effectively promote the coordinated development of industrialization and ecological environment in Anhui section of Huaihe River Economic Belt, and reduce the excessive dependence of economic growth on resources, has become an urgent practical problem to be solved. Industrial eco-efficiency, which takes into account the dual attributes of industrial development and ecological protection, has become a key indicator to evaluate the coupling coordination between industrial development and ecological environment.

"Eco-efficiency" was originally proposed by Schaltegger in 1990, which measures the environmental performance of economic development by the ratio of value increment and environmental impact increment [1]. Eco-efficiency (EE) is an important indicator to reflect the level of sustainable economic growth. Super efficiency slack-based model (SSBM) and super efficiency epsilon-based model (SEBM) under meta-frontier is used to measure the eco-efficiency of 30 china's provinces [2]. In order to estimate the impact of eco-efficiency and carbon footprints on the environment, the consumption and production patterns of resource utilization in population to population and energy intensity are considered [3]. Some scholars use DEA model to calculate the eco-efficiency index of coal industry [4-5]. At present, industrial eco-efficiency is mainly studied and analyzed from three aspects: at the beginning, many scholars focused on the analysis range of specific industries, provinces and prefecturelevel cities [6-11]. Secondly, the factors influencing industrial eco-efficiency are analyzed, including the quantity and quality of foreign direct investment [12], formal and informal environmental regulations [13], fiscal decentralization [14], industrial transfer [15], etc. Thirdly, the measurement methods mainly include traditional DEA method, the combination of input- output method and DEA method, super efficiency DEA method, Malmquist index, etc. [16-19].

To sum up, scholars have carried out abundant studies on industrial eco-efficiency, but there are still some deficiencies in the existing studies. Firstly, DEA method is the most common measurement model of ecoefficiency, but at present, the measurement of industrial eco-efficiency takes less consideration of technological heterogeneity and co-evolution mechanism. Secondly, when evaluating the characteristics of industrial eco-efficiency, most of the research dimensions is relatively single, that is difficult to solve the problem of multi-input and multi-output. Therefore, this paper takes Anhui section of Huaihe River Economic Belt as the research object, starting from the actual situation of industrial economic level, energy consumption, and environmental pollution and so on, constructs the input and output index system of industrial ecoefficiency. To overcome the shortcomings of the traditional DEA method, this paper uses the undesired output SBM model and Malmquist model to measure the industrial eco-efficiency in Anhui section of Huaihe River Economic belt, and analyzes its temporal-spatial evolution characteristics and driving forces. Combined with the coupling of industrial eco-efficiency and inputoutput index in Anhui section of Huaihe River, this paper analyzes the impact of input-output on industrial eco-efficiency. Based on Tobit regression model, this paper discusses the genetic mechanism of pattern evolution. It is expected to provide a basis for the comprehensive management of industrial development in Huaihe River Economic belt, and also provide a reference for the study of regional industrial eco-efficiency.

\section{Materials and Methods}

\section{Study Area}

The Anhui section of Huaihe River Economic Belt, locates in the interior of eastern China, is a crucial energy base of Yangtze River Delta economic zone, a link between eastern and western regions, and a region carrying on industrial transfer from the developed coastal areas. Statistics show that in 2018, the resident population of the basin was 17.578 million, accounting for $68.15 \%$ of Anhui Province; The GDP was 1158.99 billion yuan, accounting for $78 \%$ of Anhui Province; The per capita GDP reached 34092 yuan, and the fiscal revenue was 95.05 billion yuan, accounting for $18 \%$ of the province; The industrial added value was 436.83 billion yuan, accounting for $32 \%$ of Anhui Province. The industrial development in Anhui section of Huaihe River Economic Belt is one of the important driving forces for the high-quality development of the basin economy, and it can also promote the rapid development of social economic in Anhui Province. 


\section{Research Method}

\section{SBM-Undesirable Model}

The SBM-Undesirable model can not only effectively solve the problem of input variable crowding or relaxation, but also measure the eco-efficiency with "undesirable output" and "negative output" indicators, which makes up for the radial and angular deviation of the DEA model [20-21]. Therefore, this paper uses SBM-Undesirable model to measure the industrial ecoefficiency of Anhui section of Huaihe River Economic Belt, in order to ensure that the estimated value of industrial eco-efficiency is more scientific and effective. For $n$ DMUs, each DMU has $m$ inputs of $x, S_{1}$ kinds of desirable outputs of $\mathrm{y}^{\mathrm{g}}$ and $S_{2}$ kinds of undesirable outputs of $\mathrm{y}^{\mathrm{b}}$, and the vector is expressed as $x \in R^{m}, y^{g}$ $\in R^{S_{1}}, y^{b} \in R^{S_{2}}$.

$$
\begin{aligned}
& \rho^{*}=\left.\min \frac{1-\frac{1}{\mathrm{~m}} \sum_{i=1}^{m} s_{i=1}^{-} / x_{i 0}}{1+\frac{1}{s_{1}+s_{2}}\left(\sum_{i=1}^{s_{1}} s_{i}^{g} / y_{i 0}^{g}+\sum_{i=1}^{s_{2}} s_{i}^{b} / y_{i 0}^{b}\right.}\right) \\
& \text { s.t. }\left\{\begin{array}{l}
x_{0}=X \lambda+S^{-} \\
y_{0}^{\mathrm{g}}=Y^{g} \lambda-S^{g} \\
y_{0}^{\mathrm{b}}=Y^{b} \lambda+S^{b} \\
S^{-} \geq 0, S^{g} \geq 0, S^{b} \geq 0, \lambda \geq 0
\end{array}\right.
\end{aligned}
$$

In formula (1), $S^{-}, S^{g}, S^{b}$ are slack variables of input, desirable output and undesirable output respectively; $\lambda$ represents the weight vector; The target function $\rho^{*}\left(0 \leq \rho^{*} \leq 1\right)$ is strictly decreasing with respect to $S^{-}, S^{g}$, $S^{b}$. When $S^{-}=S^{g}=S^{b}=0$, that is $\rho^{*}=1$, the DMU is effective, that is, the optimal combination of input and output is achieved. When one of the three $S^{-}, S^{g}, S^{b}$ is not equal to 0 , that is $0 \leq \rho^{*} \leq 1$, the DMU is invalid, that is, the input-output ratio needs to be further optimized.

Based on the existing research results [22-24], this paper divides the calculation results of industrial ecoefficiency into four levels by using the non-equidistant division method. $0 \sim 0.5$ is the low efficiency, $0.5 \sim 0.7$ is the intermediate efficiency, $0.7 \sim 0.9$ is the good efficiency and $0.9 \sim 1.0$ is the high quality efficiency. Both good efficiency and high quality efficiency belong to high efficiency.

\section{Malmquist Productivity Index}

Malmquist productivity index can be used to measure the dynamic change of production efficiency index. If Malmquist index is greater than 1, it indicates that the current production efficiency has improved compared with the previous period.

$$
\begin{gathered}
M_{0}^{\mathrm{t}, t+1}=E F F C H^{t, t+1} \times T E C H C H^{t, t+1} \\
E F F C H^{\mathrm{t} t+1}=\frac{D_{0}^{t+1}\left(x_{t+1}, y_{t+1}\right)}{D_{0}^{t}\left(x_{t}, y_{t}\right)} \\
T E C H C H^{\mathrm{t}, t+1}=\sqrt{\frac{D_{0}^{t}\left(x_{t+1}, y_{t+1}\right)}{D_{0}^{t+1}\left(x_{t+1}, y_{t+1}\right)}} \times \sqrt{\frac{D_{0}^{\mathrm{t}}\left(x_{t}, y_{t}\right)}{D_{0}^{t+1}\left(x_{t}, y_{t}\right)}} \\
M_{0}^{t, t+1}\left(x_{t}, y_{t}, x_{t+1}, y_{t+1}\right) \\
\times \sqrt{\frac{D_{0}^{t+1}\left(x_{t+1}, y_{t+1}\right)}{D_{0}^{t}\left(x_{t+1}, y_{t+1}\right)}} \times \sqrt{\frac{D_{0}^{t}\left(x_{t}, y_{t}\right)}{D_{0}^{t+1}\left(x_{t+1}, y_{t+1}\right)}}
\end{gathered}
$$

In formula (2), $\left(x_{t}, y_{t}\right),\left(x_{t+1}, y_{t+1}\right)$ represents the input and output variables of $t$ period and $t+1$ period; $D_{0}^{t}\left(x_{t+1}, y_{t+1}\right)$ means that the technical level index of the period $t$ is used to calculate the efficiency value of the period $t+1 ; D_{0}^{t+1}\left(x_{t+1}, y_{t+1}\right)$ indicates that the ecoefficiency of the current period is indicated by the ${ }^{t+1}$ period, $M_{0}^{\mathrm{t}, t+1}$ denotes the efficiency value in the period from $t$ to $t+1$ under ecological environment constraints. $E F F C H^{\mathrm{t}, t+1}$ represents the maximum possible approximation level to the production frontier from $t$ period to $t+1$ period, so as to weigh whether the industrial production efficiency of DMU is closer to the current production frontier; $E F F C H^{\mathrm{t}, t+1}$ indicates the degree of catch-up in the technological frontier from $t$ to $t+1$. If it is greater than 1 , there is technological progress [25].

\section{Indicator Selection and Data Source}

The purpose of index selection is to characterize the interactive relationship among regional input, desirable output and undesirable output, and comprehensively reflect the regional ecological efficiency. Referring to the relevant research results, combined with the actual industrial situation and ecological endowment differences of eight cities in Anhui section of Huaihe River Economic Belt, the industrial eco-efficiency is divided into three levels: input, desirable output and undesirable output, as shown in Table 1. The fixed increase value of industry is regarded as capital input, and the number of employees in the secondary industry is regarded as labor input. Industrial resources are the basic elements of enterprise operation and development, and the total demand of industrial resources is regarded as natural resource input. Industrial added value above scale is the ultimate target output of enterprises, which can be regarded as the desirable output. Industrial waste water, waste gas, smoke and dust, solid waste and so on are the nonessential outputs accompanying the target products, which are regarded as the non-desired outputs.

Considering the real-time, consistency and accessibility of the research data, the original data of 
Table 1. Index system of industrial eco-efficiency.

\begin{tabular}{|c|c|c|c|}
\hline Target layer & Criterion layer & Index layer & Unit \\
\hline \multirow{4}{*}{ Input } & Capital input & Total industrial fixed assets & 100 million yuan \\
\cline { 2 - 4 } & Labor input & $\begin{array}{c}\text { Number of people employed in the secondary } \\
\text { industry }\end{array}$ & ten thousand people \\
\cline { 2 - 4 } & Natural resources input & Total demand for industrial resources & Ten thousand tons standard coal \\
\hline \multirow{3}{*}{ Desirable output } & Industrial output & Industrial added value above designated size & 100 million yuan \\
\hline \multirow{3}{*}{ Undesirable output } & Water pollution & Industrial Waste Water Discharged & Ten thousand tons \\
\cline { 2 - 4 } & Atmospheric pollution & Industrial $\mathrm{SO}_{2}$ emissions & ton \\
\cline { 2 - 4 } & Solid waste emissions & Industrial smoke and dust emissions & Ten thousand tons \\
\cline { 2 - 4 } & & Industrial solid waste production & Ten \\
\hline
\end{tabular}

the indicators involved in this paper are all from the Statistical Yearbook of Anhui Province (2010-2019), the Statistical Yearbook (2010-2019) and Statistical Bulletin (2010-2018) of eight cities in Anhui section of the Huaihe River Economic Belt, and the official website of the Environmental Protection Bureau, etc.

\section{Results and Discussion}

\section{Time Series Characteristics of Industrial Eco-Efficiency}

According to the formula (1), the industrial ecoefficiency of eight cities in Anhui section of Huaihe River Economic Belt from 2009 to 2018 was calculated. Among them, Huaibei, Bozhou, Suzhou, Bengbu, Fuyang and Huainan belonged to northern Anhui; Chuzhou and Lu'an pertained to central Anhui. The calculation results showed that the overall industrial eco-efficiency of eight cities in Anhui section of Huaihe River Economic Belt was 0.717, which was in the middle level. The eco-efficiency was at the good level but closed to the intermediate level. From the change in trend (Fig. 1), the overall industrial eco-efficiency

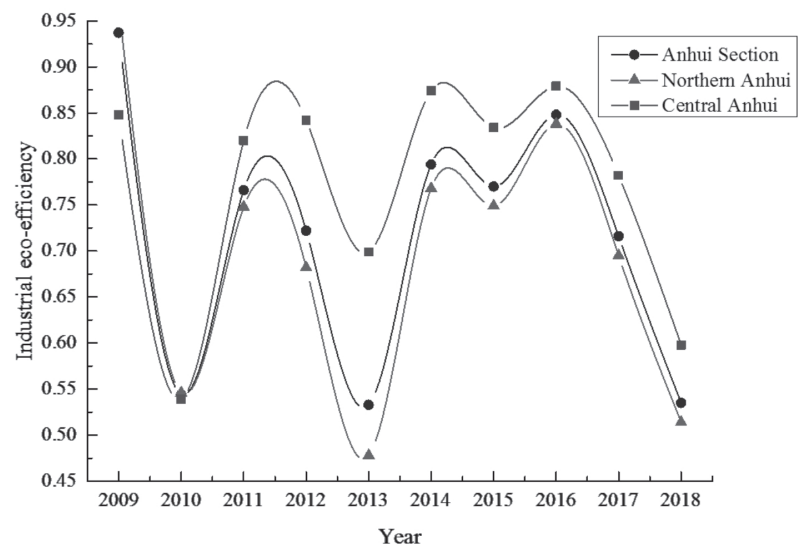

Fig. 1. Industrial eco-efficiency value from 2009 to 2018. of Anhui section decreased in the fluctuation from 2009 to 2018, with an average annual growth rate of $-1.976 \%$, and the fluctuation range was small. During the study period, the comprehensive industrial ecoefficiency in Anhui section of Huaihe River Economic Belt reached the maximum value of 0.937 in 2009 and dropped to the lowest value of 0.533 in 2013. The main reasons for this change were that industrial enterprises still consumed coal resources as the main energy, the industrial structure was seriously unreasonable, and the growth rate of high-tech industries and strategic emerging industries was low, which restricted the benign balance between industrial development and ecological construction. From the regional perspective, the industrial eco-efficiency of central Anhui was the highest, which decreased significantly in 2010; gradually picked up from 2011 to 2017, and showed a stable curve change. In 2018, the industrial eco-efficiency showed a "cliff" decline. As a whole, the industrial eco-efficiency in Northern Anhui was lower than that in Anhui, and the fluctuation characteristics were obvious, and the fluctuation range was gradually increasing. This change also caused the sharp fluctuation on the average value of the whole Anhui section. The reason was that the resource-based cities in Northern Anhui were in the critical period of industrial transformation and upgrading, even though the investment in infrastructure and technological transformation was increasing. However, it was difficult for the relevant enterprises to adapt to the new market environment formed by the industrial transformation quickly, which affected the enterprise benefits and leaded to the reduction of industrial efficiency. This also indicated that there was a great space to improve the industrial eco-efficiency in Northern Anhui.

The dynamic evolution trend of industrial ecoefficiency of Anhui section of Huaihe River Economic Belt in 2008, 2013 and 2018 was drawn by using the kernel density function estimation method (Fig. 2). It could be seen from Fig. 2 that in 2009 and 2018, the center of industrial eco-efficiency kernel density curve showed a trend of right deviation. While in 2013, 


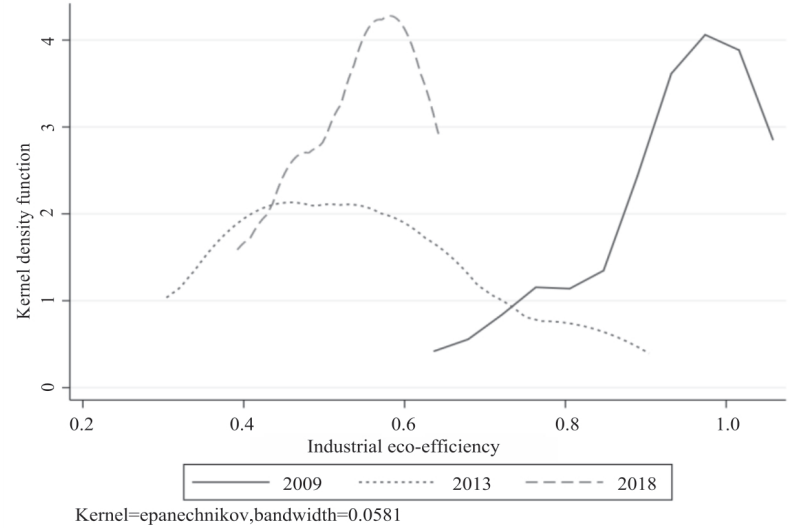

Fig. 2. Kernel density curve of industrial eco-efficiency in 2009, 2013 and 2018.

it presented a trend of left deviation, which indicated that the concentration range of industrial eco-efficiency showed an unstable change trend. The change range of the curve increased first and then decreased, indicating that the overall gap of industrial eco-efficiency had expanded. Compared with 2009, the "peak" of the curve in 2018 was steeper, and the peak value was slightly higher, which indicated that the gap of industrial ecoefficiency among prefecture level cities in the study area had a widening trend. Compared with 2009, the "peak" of the curve in 2013 was much slower, and the peak value decreased greatly, which indicated that the gap of industrial eco-efficiency among prefecture level cities had a significant narrowing trend. In 2018, the value of industrial eco-efficiency was between 0.4 and 0.7 , with a low overall level. Therefore, the cooperation among cities should be strengthened to promote the transformation and upgrading of industrial structure in low-efficiency regions and further enhance the regional industrial eco-efficiency.

\section{Spatial Characteristics of Industrial Eco-Efficiency}

(1) Spatial pattern analysis of industrial eco-efficiency Industrial eco-efficiency not only has the characteristics of time series evolution, but also shows the regional differences of spatial pattern [26]. In order to make a more intuitive analysis about the changes of
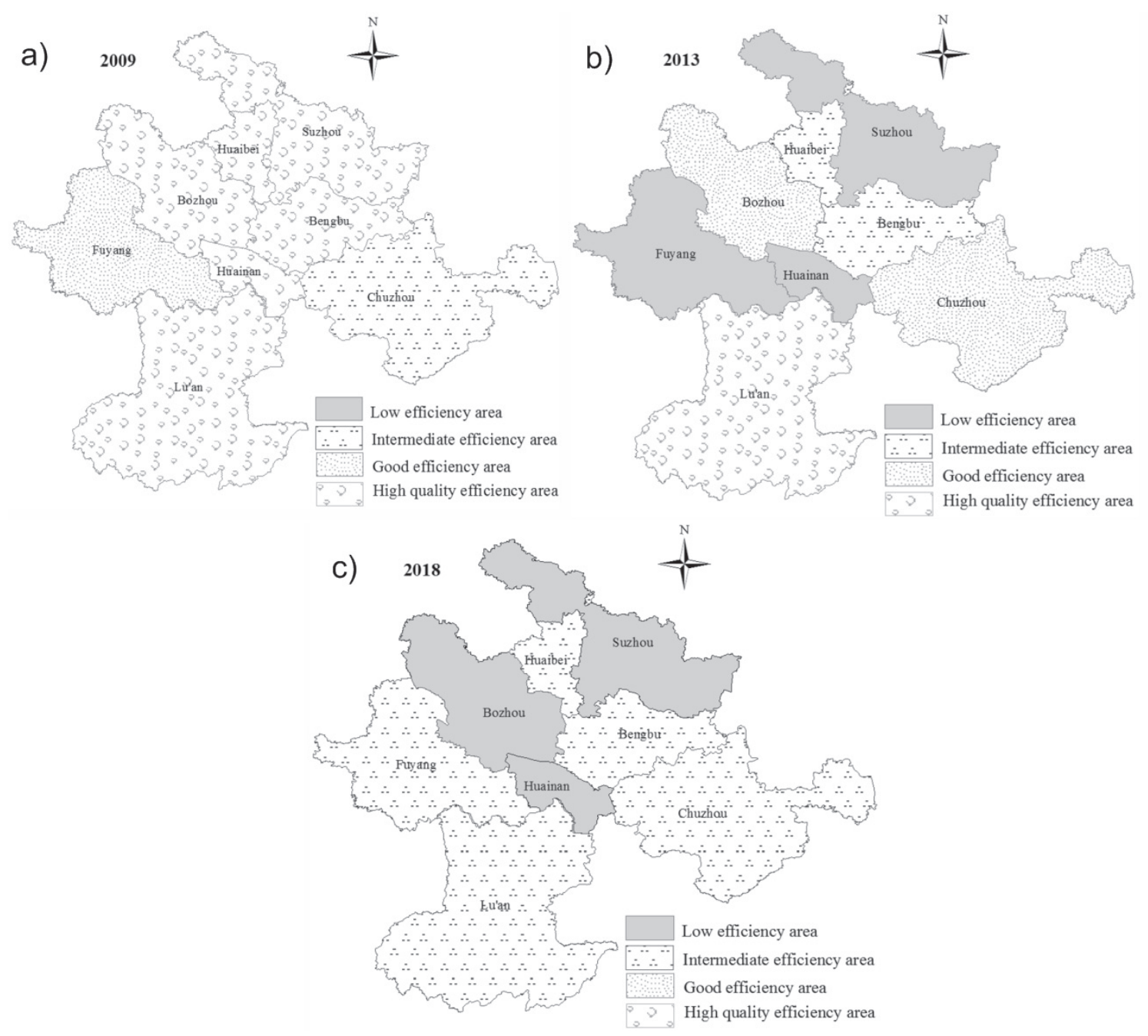

Fig. 3. Spatial distribution pattern of industrial eco-efficiency in a) 2009, b) 2013 and c) 2018 . 
industrial eco-efficiency in Anhui section of Huaihe River Economic Belt from 2009 to 2018, this paper used ArcGIS10.2 software and natural discontinuity method to visually analyze the spatial pattern of industrial ecoefficiency in 2009, 2013 and 2018 (Fig. 3). What's more, the spatial trend curve of industrial eco-efficiency was drawn by using trend analysis tool (Fig. 4), where the direction indicated by $\mathrm{x}$-axis was due east, and the direction indicated by $y$-axis was due north.

It could be seen from Fig. 3 that during the study period, the spatial distribution characteristics of industrial eco-efficiency in Anhui section of Huaihe
River Economic Belt were relatively significant. From the changes of cities at each level, the number of cities with four levels of efficiency had changed. In 2009, the number of cities corresponding to low - intermediate good - high quality efficiency was 0-1-1-6. In 2013, the number of cities corresponding to low - intermediate good - high quality was 3-2-2-1. In 2018, the number of cities corresponding to low - intermediate - good - high quality was 3-5-0-0 respectively. From the perspective of spatial distribution, the distribution pattern of industrial eco-efficiency in Anhui section of Huaihe River Economic Belt in 2009 showed obvious

a)

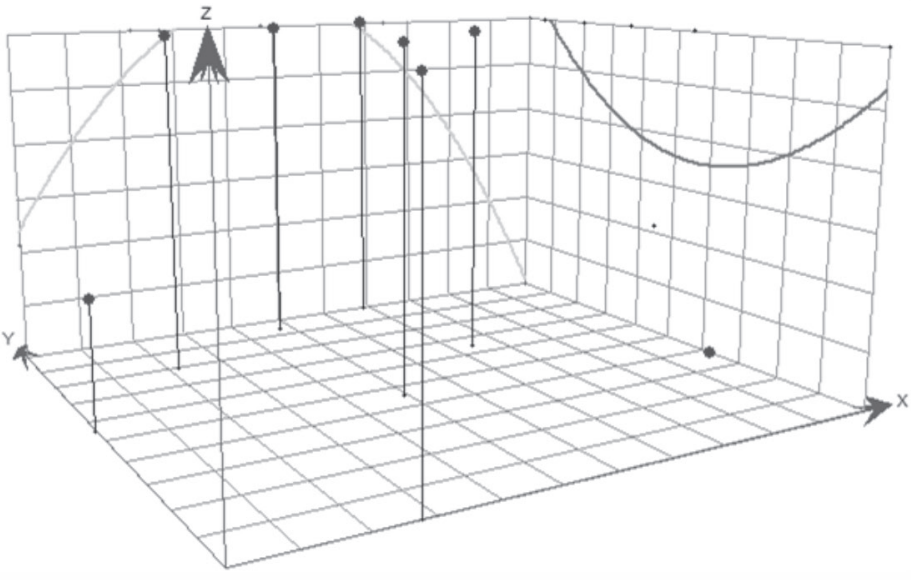

b)

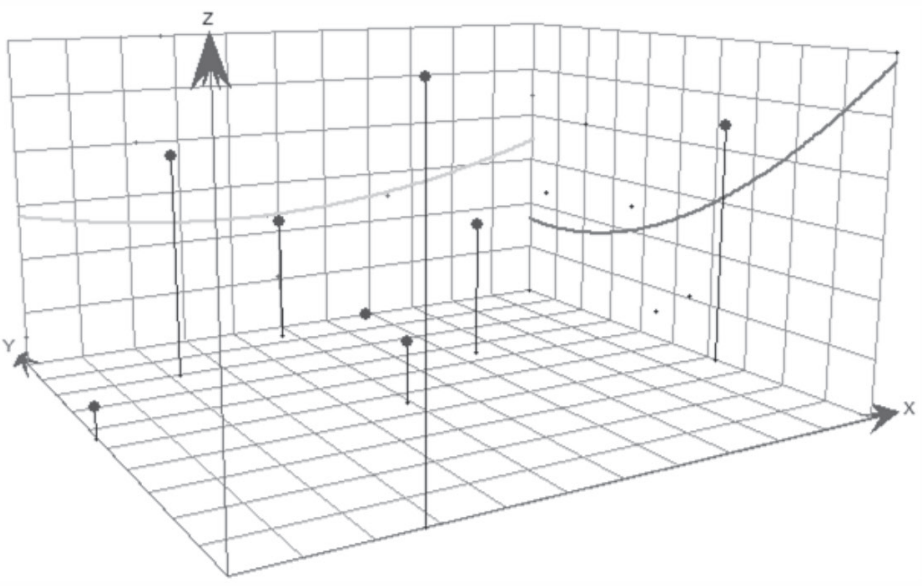

c)

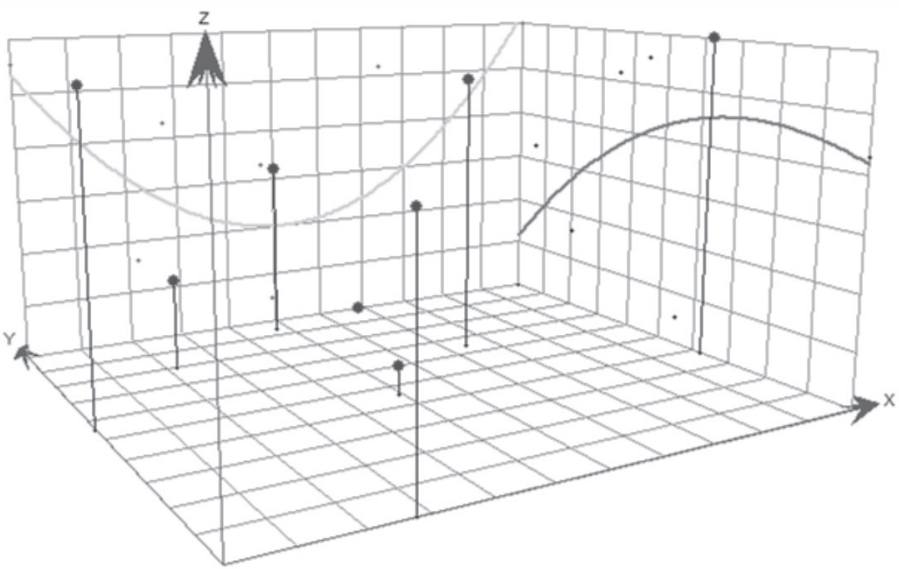

Fig. 4. Global trend chart of industrial eco-efficiency in a) 2009, b) 2013 and c) 2018. 
agglomeration characteristics, that was, the "southnorth" industrial eco-efficiency was high quality, while the "east-west" industrial eco-efficiency level was relatively low. In 2013, high quality efficiency and good efficiency cities were mainly concentrated in central Anhui (Lu'an and Chuzhou). While industrial eco-efficiency in Northern Anhui was generally low, especially in Huainan and Suzhou, which decreased from the high quality efficiency level in 2009 to the low efficiency level in 2013. In 2018, the whole research area was downgraded to low and medium efficiency level. Furthermore, the original spatial pattern was broken.

According to the global trend chart of industrial eco-efficiency in Fig. 4, the industrial eco-efficiency of eight cities in Anhui section of the Huaihe River Economic Belt had changed from an inverted "U" shaped structure with "low at both ends and high in the middle" in 2009 to a "U" shaped structure with "low in the middle and high at both ends" in 2018. In the northsouth direction, the industrial eco-efficiency changed from "U" structure to a gradient spatial pattern of "high in the South and low in the north", and the curvature of the curve became slightly flat, which indicated that the industrial eco-efficiency in the southeast of Anhui section of Huaihe River Economic Belt had improved significantly, and the industrial eco-efficiency in the northern of Anhui had become the weak area of Anhui section.

(2) Spatial correlation analysis of industrial ecoefficiency

The spatial distribution of industrial eco-efficiency was characterized by a concentrated and contiguous "block" distribution, and the changes of eco-efficiency among regions might also have spatial correlation. The global Moran index of industrial eco-efficiency on Anhui section of Huaihe River Economic Belt from 2009 to 2018 was calculated by using GeoDa software (Fig. 5), and its spatial heterogeneity and dependence were analyzed.

As can be seen from Fig. 5, the industrial ecoefficiency in Anhui section of Huaihe River Economic

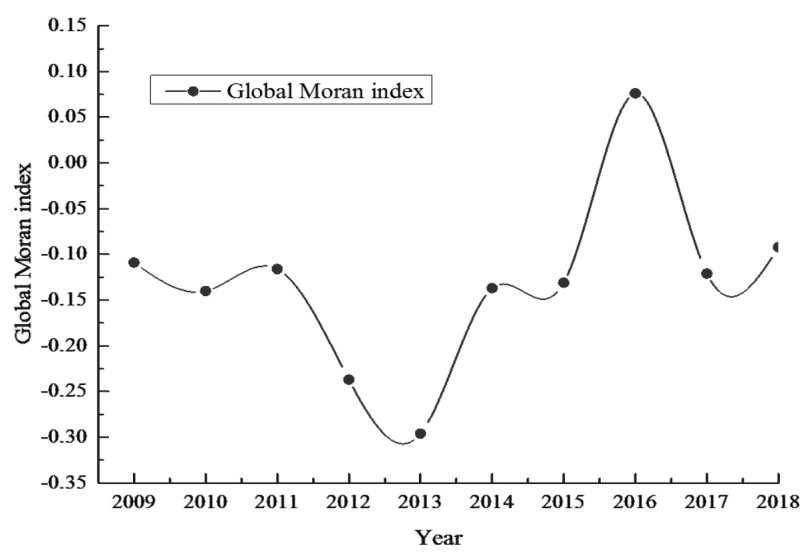

Fig. 5. Global Moran index of industrial eco-efficiency from 2009 to 2018.
Belt fluctuated within the interval of $[-0.3,0.08]$, and was significant at the level of $10 \%$, which indicated that the spatial distribution of industrial eco-efficiency was not random, and there was a strong spatial dependence among different cities. From 2009 to 2011, the global Moran index of industrial eco-efficiency tended to be stable, fluctuated around -0.10 , and the spatial impact of industrial eco-efficiency continued to exist. The global Moran index of industrial eco-efficiency decreased sharply from 2011 to 2013, and reached the lowest value of -0.296 in 2013. Moran's $\mathrm{I}<0$ indicated negative spatial correlation, and the smaller the value was, the greater the spatial difference was. The global Moran index continued to increase from 2013 to 2016, and the spatial agglomeration degree of industrial ecoefficiency reached the peak in 2016. The global Moran index was 0.076 . Moran's I $>0$ indicated that the spatial agglomeration was positively correlated with small spatial differences.

(3) Industrial eco-efficiency and input-output coupling

Industrial eco-efficiency was affected by the comprehensive relationship of input, desired output and undesired output. Therefore, in order to analyze the relationship between industrial eco-efficiency and inputoutput in Anhui section of Huaihe River Economic Belt, and to analyze the differences of different combination types of industrial eco-efficiency and input-output, this paper made an in-depth study and analysis about the impact of input, desired output and undesired output on industrial eco-efficiency.

Combined with the academic research [27], firstly, the input and output indicators were dimensionless and standardized. Secondly, it was weighted to make the index range of each index between 0 and 1 . Then, the input-output index was coupled with the industrial eco-efficiency. Finally, the equal weight assignment method was used to calculate the input-output index. By referring to the relevant research [28], and combining with the classification standard of industrial ecoefficiency in Anhui section, it could be divided into three types: low efficiency $0 \sim 0.5$, medium efficiency $0.5 \sim 0.7$ and high efficiency 0.7 1. According to the results of input and output index, there were 10 inputoutput types of industrial eco-efficiency in the study area. By superimposing the input-output type with the efficiency type of Anhui section, the coupling relationship between industrial eco-efficiency and input-output could be obtained, as shown in Table 2.

As can be seen from Table 2, the coupling types of industrial eco-efficiency and input-output in Anhui section of the Huaihe River Economic Belt in 2009, 2013 and 2018 were different. In 2009, the industrial eco-efficiency at the high level was divided into four types. The cities with "medium efficiency, low consumption and low pollution" accounted for a large proportion, mainly including Bengbu, Suzhou and Lu'an. Huainan and Huaibei were in the forefront of efficiency, belonging to the development mode I and II. The economic benefits of the two cities had increased 
Table 2. Coupling relationship between industrial eco-efficiency and input-output in Major. Years.

\begin{tabular}{|c|c|c|c|c|c|c|c|}
\hline Year & Type & & Pattern & & Low efficiency & Medium efficiency & High efficiency \\
\hline \multirow{7}{*}{2009} & I & High benefit & $\begin{array}{c}\text { Medium } \\
\text { consumption }\end{array}$ & High pollution & & & Huainan \\
\hline & II & High benefit & $\begin{array}{c}\text { Medium } \\
\text { consumption }\end{array}$ & Low pollution & & & Huaibei \\
\hline & III & $\begin{array}{l}\text { Medium } \\
\text { benefit }\end{array}$ & Low consumption & Low pollution & & & $\begin{array}{l}\text { Bengbu, Suzhou, } \\
\text { Lu'an }\end{array}$ \\
\hline & IV & $\begin{array}{l}\text { Medium } \\
\text { benefit }\end{array}$ & $\begin{array}{c}\text { Medium } \\
\text { consumption }\end{array}$ & Low pollution & & & Fuyang \\
\hline & V & $\begin{array}{l}\text { Medium } \\
\text { benefit }\end{array}$ & $\begin{array}{c}\text { Medium } \\
\text { consumption }\end{array}$ & $\begin{array}{l}\text { Medium } \\
\text { pollution }\end{array}$ & & Chuzhou & \\
\hline & VI & Low benefit & Low consumption & Low pollution & & & Bozhou \\
\hline & I & High benefit & $\begin{array}{c}\text { Medium } \\
\text { consumption }\end{array}$ & High pollution & Huainan & & \\
\hline \multirow{5}{*}{2013} & II & High benefit & $\begin{array}{c}\text { Medium } \\
\text { consumption }\end{array}$ & Low pollution & & Bengbu & \\
\hline & III & $\begin{array}{l}\text { Medium } \\
\text { benefit }\end{array}$ & Low consumption & Low pollution & & & Lu'an \\
\hline & IV & $\begin{array}{l}\text { Medium } \\
\text { benefit }\end{array}$ & $\begin{array}{c}\text { Medium } \\
\text { consumption }\end{array}$ & Low pollution & $\begin{array}{l}\text { Fuyang, } \\
\text { Suzhou }\end{array}$ & & \\
\hline & $\mathrm{V}$ & Low benefit & Low consumption & Low pollution & & & Bozhou \\
\hline & VI & High benefit & $\begin{array}{c}\text { Medium } \\
\text { consumption }\end{array}$ & $\begin{array}{l}\text { Medium } \\
\text { pollution }\end{array}$ & Chuzhou & Huaibei & \\
\hline \multirow{6}{*}{2018} & I & High benefit & $\begin{array}{c}\text { Medium } \\
\text { consumption }\end{array}$ & Low pollution & & Bengbu, Chuzhou & \\
\hline & II & $\begin{array}{c}\text { Medium } \\
\text { benefit }\end{array}$ & $\begin{array}{c}\text { Medium } \\
\text { consumption }\end{array}$ & $\begin{array}{l}\text { Medium } \\
\text { pollution }\end{array}$ & & Fuyang & \\
\hline & III & Low benefit & Low consumption & Low pollution & Bozhou & Lu'an & \\
\hline & IV & Low benefit & $\begin{array}{c}\text { Medium } \\
\text { consumption }\end{array}$ & High pollution & Huainan & & \\
\hline & V & Low benefit & $\begin{array}{c}\text { Medium } \\
\text { consumption }\end{array}$ & Low pollution & Suzhou & & \\
\hline & VI & Low benefit & Low consumption & $\begin{array}{l}\text { Medium } \\
\text { pollution }\end{array}$ & & Huaibei & \\
\hline
\end{tabular}

the value of industrial eco-efficiency. Bozhou belonged to the type of VI, with low resource consumption, low industrial economic output and environmental damage. Fuyang belonged to "medium benefit, medium consumption and low pollution", which could improve efficiency value by reducing environmental pollution. Different from Fuyang, although it had the same economic level and energy consumption, Chuzhou had caused serious environmental pollution in the process of development, so its eco-efficiency was at a medium level.

In 2013, the level of high efficiency was only Lu'an and Bozhou, with the same type of input and output. The industrial economic output of Huainan, Huaibei and Chuzhou was high, but the resource consumption and ecological environment pollution were serious in the process of development, so the industrial ecoefficiency was not high. The increase of resource consumption in Bengbu and Suzhou leaded to the decline of industrial eco-efficiency. In 2018, the industrial eco-efficiency of Lu'an and Bozhou was relatively ineffective. The reason was that although the resource consumption and environmental pollution of this type were relatively low, their economic output was also relatively low, which leaded to low improvement of residents' welfare. Therefore, the overall efficiency was at a low level. The environmental pollution and ecological damage in Huainan and Huaibei were serious, which had restricted the economic development, while other cities had little change. From 2009 to 2018, most of the eight cities in Anhui section were in the categories of II and VI, while a few were in category VII-X. The characteristics of "high efficiency and high pollution" type cities were obvious, mainly in Huainan and Huaibei. This also showed that most cities still stay in the traditional industrial development mode, 


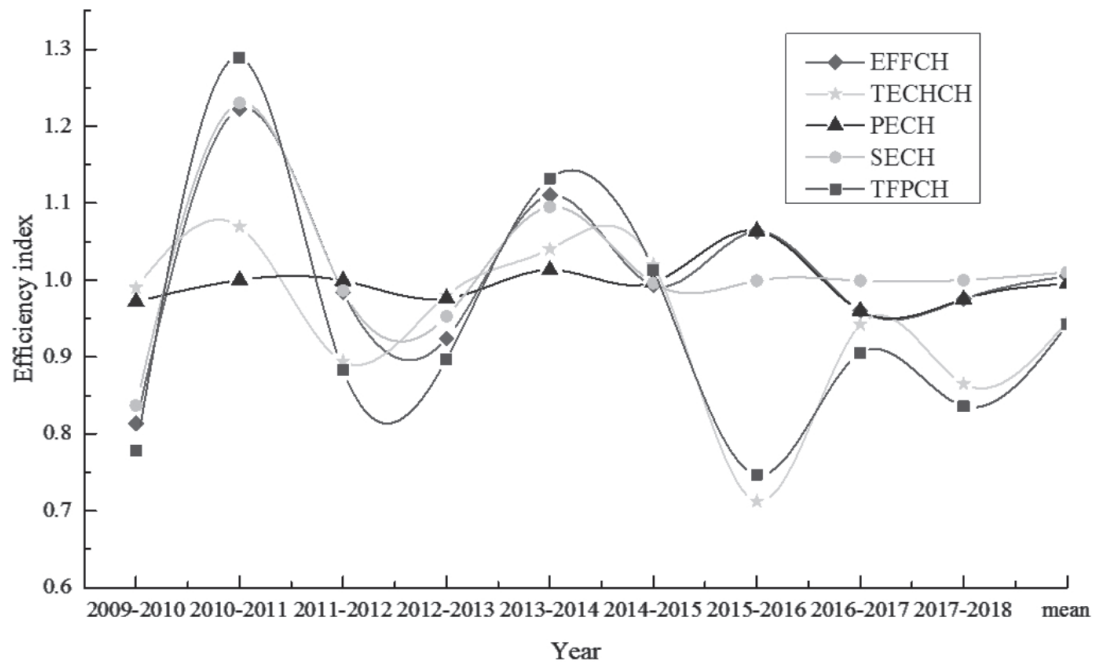

Fig. 6. Malmquist index decomposition of industrial eco-efficiency.

and the level of industrial production efficiency was low.

In addition, even in the same type of input-output development, the characteristics of industrial ecoefficiency also showed significant differences. For example, in 2018, Bozhou and Lu'an were both in the VI input-output model, but the performance of ecological efficiency in this type of city was different. Lu'an was in the medium efficiency stage, while Bozhou was in the low efficiency stage, which might be due to the difference in the relationship between output level and input level ratio. When the input was less than the desired output and greater than the undesired output, the regional industrial eco-efficiency was generally at a higher level of development.

\section{Dynamic Evolution of Industrial Eco-Efficiency}

In order to deeply analyze the dynamic change characteristics and driving factors of industrial ecoefficiency in Anhui section of Huaihe River Economic Belt, the Malmquist index was used to calculate the dynamic evolution trend of industrial eco-efficiency from 2009 to 2018. As shown in Fig. 6, in the research period, the average value of total factor productivity (TFPCH) was 0.942 , and the average annual growth rate was - 5.8\%, which indicated that the level of industrial eco-efficiency in Anhui section was declining in fluctuation, and the fluctuation range was large. The average value of the technical efficiency index $(\mathrm{EFFCH})$ was 1.005 , with an average annual growth rate of $0.5 \%$. The change trend of the technical efficiency index from 2009 to 2015 was basically consistent with the total factor productivity index, and the technical efficiency index had a small increase from 2015 to 2018, which indicated that the technical efficiency index was the leading force to promote the improvement of TFPCH. The technology progress index (TECHCH) showed a fluctuating downward trend, with an average value of 0.946 and an average annual growth rate of $-5.4 \%$. It showed that the technological progress index of industrial frontier production in Anhui section was an important reason for lowering $\mathrm{TFPCH}$ index. It failed to follow a good production mode in the development of industrial production and fell behind the growth rate of technical efficiency index as a whole. The average value of pure technical efficiency index $(\mathrm{PECH})$ was 0.995 , and its fluctuation range from 2009 to 2014 was small, which indicated that the overall level of technology management and technology application level of industrial enterprises were slow to catch up with the production frontier without considering the impact of scale efficiency. Therefore, the level of industrial technology management in Anhui Section needs to be further improved in the future. From the perspective of scale efficiency (SECH) index change, the average change index of the scale efficiency of industrial eco-efficiency in Anhui section from 2009 to 2018 was 1.010, which was consistent with the change trend of the technical efficiency index in Anhui section during 2009-2014, and showed a trend of decreasing first and then rising during 2014-2018. The fluctuation range was small, which indicated that the difference between the industrial production scale and the optimal production scale was small in Anhui section.

The TFPCH index and its decomposition of industrial eco-efficiency of eight cities in Anhui section from 2009 to 2018 were shown in Table 3. On the whole, the average TFPCH index of industrial eco-efficiency of eight cities in the study period was less than 1 except that of Lu'an. The TFPCH index of industrial eco-efficiency in Lu'an had achieved positive growth. The reason was that the level of industrial eco-efficiency in Lu'an was low and there was a large space for improvement. Therefore, the improvement speed was faster than that in other high-level areas. 
Table 3. Malmquist index of industrial eco-efficiency and its decomposition.

\begin{tabular}{|c|c|c|c|c|c|}
\hline City & EFFCH & TECHCH & PECH & SECH & TFPCH \\
\hline Bengbu & 0.981 & 0.998 & 0.975 & 1.023 & 0.970 \\
\hline Huainan & 1.000 & 0.827 & 1.000 & 1.000 & 0.827 \\
\hline Fuyang & 1.043 & 0.935 & 1.003 & 1.031 & 0.946 \\
\hline Lu'an & 1.004 & 1.011 & 1.001 & 1.001 & 1.023 \\
\hline Bozhou & 1.000 & 0.946 & 1.000 & 1.000 & 0.946 \\
\hline Suzhou & 1.037 & 0.923 & 1.008 & 1.029 & 0.915 \\
\hline Huaibei & 1.000 & 0.954 & 1.000 & 1.000 & 0.955 \\
\hline Chuzhou & 0.973 & 0.970 & 0.974 & 0.998 & 0.955 \\
\hline
\end{tabular}

The increase of TFPCH index was mainly due to the rise of EFFCH and TECHCH index. From 2009 to 2012, the EFFCH index continued to rise and remained at 1 after 2012. The TECHCH index rose as a whole in the study period, with a small fluctuation. Therefore, the process production mode of enterprises in Lu'an was continuously optimized and improved, which promoted the development of industrial eco-efficiency.

Secondly, the TFPCH indexes of Bengbu, Fuyang, Bozhou, Suzhou, Huaibei and Chuzhou all decreased in different proportions, ranging from $3 \%$ to $8.5 \%$. The average annual growth rate of Bengbu's TFPCH index was $-3 \%$. The reason was that the TECHCH and EFFCH indexes decreased at the same time. During the study period, The TECHCH index decreased by $0.2 \%$ and the EFFCH index decreased by $1.9 \%$. Due to its large economic size, industrial transformation was difficult, which affected the improvement of industrial eco-efficiency. Fuyang and Bozhou have the same degree of decline, both of which were $5.4 \%$. The reason for the decline was the effect of TECHCH index, which indicated that technological progress was the most important factor affecting the industrial eco-efficiency of the two cities. Similarly, the decline of TFPCH index in Suzhou was mainly caused by the change of TECHCH index, and the technological progress index showed negative growth in most years of the study period. Huaibei and Chuzhou have the same degree of decline, both of which are $4.5 \%$. The decline of TFPCH index in Huaibei was unilaterally caused by the decline of TECHCH index. The technological progress index declined after 2012, which pulled down the TFPCH index of industrial eco-efficiency in Huaibei. However, the decline of TFPCH index of Chuzhou was due to the dual influence of EFFCH and TECHCH index. Therefore, Chuzhou should not only speed up the industrialization process, but also pay attention to the new technological innovation. The TFPCH index in Huainan area decreased fastest, with an average annual decrease of $17.3 \%$. It can be seen that $\mathrm{TECHCH}$ was the main reason for the decrease of TFPCH index. From 2009 to 2017, the TECHCH index of industrial ecoefficiency in Huainan showed a trend of first rising and then declining, while from 2017 to 2018, the TECHCH index showed a small increase. Generally speaking, the decline of TECHCH index seriously hindered the development of eco-efficiency in Huainan.

By contrast, except Bengbu (0.975) and Chuzhou (0.974), the average PECH index of eight cities in Anhui section of Huaihe River Economic Belt from 2009 to 2018 was greater than or equal to 1 . Except Chuzhou (0.998), the average value of SECH index was greater than or equal to 1 . The PECH and SECH index values were generally good, which indicated that the industrial production management level of Anhui section was close to the forefront of industrial production, but the level of industrial technology management and technology application should be continued to be improved in the future. At present, the gap between the industrial production scale and the optimal scale in most cities is narrowing. Therefore, optimizing the allocation of resources and reasonably adjusting the layout can effectively promote the industrial eco-efficiency of Anhui section.

\section{Analysis on the Influencing Factors of Industrial Eco-Efficiency}

The promotion and improvement of industrial eco-efficiency in Anhui section of Huaihe River Economic Belt are affected by many factors. According to the previous calculation results, the value of industrial eco-efficiency in Anhui section of Huaihe River Economic Belt was low. Based on the actual industrial situation of the region, referring to the relevant literature research and considering the actual availability of data, six aspects were finally determined as the main influencing factors of industrial eco-efficiency in Anhui section of Huaihe River Economic Belt, and Tobit regression model was used to quantitatively analyze the influencing factors [29-30]. Among them, the impact direction of economic development level (RGDP) on industrial eco-efficiency was the result of the game between positive and negative externalities, and the per capita GDP was used to represent the level of economic development. Urbanization level (UR) was an important 
Table 4. Tobit regression results.

\begin{tabular}{|c|c|c|c|}
\hline Variable & $\begin{array}{c}\text { Parameter } \\
\text { estimation }\end{array}$ & Z statistic & P value \\
\hline RGDP & $0.449^{* * *}$ & 2.720 & 0.008 \\
\hline UR & $-0.857^{* * *}$ & -4.490 & 0.000 \\
\hline IS & 0.005 & 1.140 & 0.257 \\
\hline OPEN & 0.010 & 0.400 & 0.689 \\
\hline RDI & $0.094^{* *}$ & 2.120 & 0.037 \\
\hline POL & $-0.117^{*}$ & -1.770 & 0.081 \\
\hline Log likelihood $=17.903$ & \multicolumn{2}{|c|}{ Prob >chi2 $=0.115$} \\
\hline
\end{tabular}

Note: $* * * * *$ and $*$ indicate significant at significance levels of $1 \%, 5 \%$ and $10 \%$, respectively

factor affecting the ecological environment and industrial development, and the urbanization rate was used to represent the level of urbanization. Industrial structure (IS) had different effects on regional energy consumption intensity and total energy consumption, and the proportion of GDP of the secondary industry was used to characterize the industrial structure. The degree of opening to the outside world (OPEN) reflected the scale and level of foreign trade in a region, and the proportion of FDI in GDP represented the degree of opening to the outside world. Research and development innovation (RDI) could improve the production process and pollution control technology, and then promoted the sustainable development of ecological environment. The proportion of R\&D internal expenditure of industrial enterprises in industrial added value was used to characterize the research and innovation. Environmental regulation (POL) played an important role in controlling environmental pollution and urging enterprises to save energy and reduce emissions, and environmental regulation was represented by the proportion of environmental protection input in government fiscal expenditure.

With the help of Stata15.1 software, the Tobit model was used to process the data. The evaluation value of industrial eco-efficiency of each city was taken as the explained variable, and the selected influencing factor was set as the explanatory variable. The specific calculation results were shown in Table 4.

The regression results showed that RGDP, RDI, UR and POL all passed the significance test, and RGDP and UR had a significant impact on the industrial eco-efficiency of Anhui section of Huaihe River Economic Belt. However, IS and OPEN didn't pass the significance test, indicating that these two factors had no obvious effect on the industrial eco-efficiency of Anhui section.

(1) The regression coefficient of economic development level to industrial eco-efficiency was 0.449 , and the significance test of $1 \%$ indicates that economic development played a role in promoting the ecological environment. On the one hand, the regional per capita GDP was closely related to the regional productivity level, technical level and labor quality, which could provide a strong guarantee for improving the industrial eco-efficiency of Anhui section of Huaihe River Economic Belt. On the other hand, the economic level of the region was at a high level. The more the total per capita income of the residents, the higher the corresponding production technology and production conditions would be. It would provide reliable material guarantee for improving the industrial eco-efficiency, and ensured to enhance the ability of enterprises to manage the industrial environment.

(2) There was a negative correlation between the level of urbanization and the industrial eco-efficiency, which indicated that the urbanization rate played a certain role in hindering the industrial eco-efficiency. The reason was that the agglomeration of urban population formed economies of scale, accelerated the development of industrialization and promoted the division of labor. However, the rapid urbanization process also leaded to the increase of water environment pollution, air pollution and solid pollution. Moreover, the ecological environment was damaged to a certain extent, and the industrial eco-efficiency was negatively affected.

(3) There was a positive correlation between industrial structure and industrial eco-efficiency, and the corresponding $\mathrm{P}$ value of the proportion of GDP of the secondary industry was 0.257 , and the regression result was not significant. This was because Anhui section of Huaihe River Economic Belt had a strong industrial foundation and large-scale industrial enterprises, which brought huge scale economy. With the further advance of supply-side structural reform, the market-oriented production capacity of iron and steel and coal industries had declined. Therefore, the economic benefits brought by the secondary industry had declined, and the impact on the regional industrial eco-efficiency had been relatively weak.

(4) The degree of opening to the outside world had a positive correlation with the industrial ecological efficiency, but the influence was not significant. Some scholars believed that foreign direct investment (FDI) could promote the improvement of eco-efficiency, while others pointed out that FDI could inhibit the improvement of eco-efficiency. In this paper, FDI had played a certain role in promoting the regional industrial eco-efficiency, but its impact had not been really played.

(5) There was a positive correlation between R\&D innovation and industrial eco-efficiency. The $\mathrm{P}$ value of the proportion of the internal $\mathrm{R} \& \mathrm{D}$ expenditure of industrial enterprises in the regional industrial added value was 0.037 , which passed the significance level test of $5 \%$, indicating that the strengthening of the R\&D and innovation ability had a positive effect on the industrial eco-efficiency in Anhui section. The stronger the ability of technological innovation, the more conducive to promote pollution reduction technology and enhance the ability of pollution controlled, and promoted the 
improvement of industrial eco-efficiency in Anhui section.

(6) There was a negative correlation between environmental regulation and industrial eco-efficiency. The index passed the significance test of $10 \%$. Strengthening environmental regulations would reduce pollution and energy consumption, but it would also lead to a reduction in GDP, which was a gradual process. In addition, the mode of pollution control after industrial development couldn't be fundamentally changed, and the effect of policy implementation lagged behind, which would affect the improvement of industrial ecoefficiency.

\section{Conclusions}

Firstly, this paper systematically expounded the temporal and spatial variation trend of industrial ecoefficiency from the perspectives of comprehensive industrial eco-efficiency, industrial eco-efficiency level of different cities and spatial pattern differences in Anhui section of Huaihe River Economic Belt. Then, the Malmquist model was used to decompose the industrial eco-efficiency, and the driving mechanism of industrial eco-efficiency was found out. Finally, the main influencing factors of industrial eco-efficiency in Anhui section were screened and the formation mechanism was analyzed, and the conclusions were as follows:

(1) The level of industrial eco-efficiency in Anhui section of Huaihe River Economic Belt was not high, and the differences of industrial eco-efficiency among the eight cities were obvious. From 2009 to 2018, the average value of industrial eco-efficiency in Anhui section was 0.717 , which was a good level, but closed to the intermediate level, and there was still much room for improvement. From the perspective of spatial distribution, the industrial eco-efficiency in Anhui section showed obvious agglomeration characteristics, showing an increasing pattern of "north to South" industrial eco-efficiency. The cities with high quality efficiency and good efficiency mostly concentrated in central Anhui, while the industrial eco-efficiency of Northern Anhui was generally low.

(2) The coupling relationship between industrial eco-efficiency and input-output showed that most of the eight cities in Anhui section of Huaihe River Economic Belt were in the input-output development mode of high benefit, medium consumption, low pollution and low benefit, low consumption and low pollution. The characteristics of "high benefit, high pollution" type cities were obvious, mainly including Huainan and Huaibei. Among them, cities in the same input-output type also showed significant differences in industrial eco-efficiency, which lied in the differences of the ratio between input level and output level in different cities.

(3) TECHCH was the most important reason for the decrease of industrial eco-efficiency in Anhui section of the Huaihe River Economic Belt. The average value of TFPCH index in Anhui Province from 2009 to 2018 was 0.942, and the TFPCH index decreased in fluctuation, with a large fluctuation range. From the perspective of decomposition index, the mean value of PECH was 0.995, which indicated that the technology management level of industrial enterprises in Anhui section was slow to catch up with the production frontier. The mean value of SECH was 1.010, indicating that the industrial production scale inAnhui section was close to the optimal scale.

(4) The influence factors had different effects on the industrial eco-efficiency. The level of economic development and $R \& D$ innovation could promote the industrial eco-efficiency in Anhui section, and play a positive role. The higher level of urbanization and environmental regulation had a restraining effect on improving the industrial eco-efficiency. The industrial structure, the degree of opening up to the outside world had no significant relationship with the industrial ecoefficiency.

\section{Acknowledgments}

This work was supported by the following programs: 1. The National Natural Science Foundation of China with the title "Evaluation of Coal Miners' Safety Behavioral Ability and Its Dynamic Pre-warning under the Interaction between Individuals and Environment" (NO. 51574010). 2. Independent Project of State Key Laboratory of Mining Response and Disaster Prevention and Control in Deep Coal Mines with the title "Research on underground dust monitoring and intelligent early warning system" (NO. SKLMRDPC20ZZ12). 3. Key Projects of Humanities and Social Sciences in Anhui with the title "Research on safety early warning system of underground support deformation monitoring based on laser intrusion detector" (NO. SK2020A0213).

\section{Conflict of Interest}

The authors declare no conflict of interest.

\section{References}

1. SCHALTEGGER S. Okologische rationalitat:Ansatzpunkte zur ausgestaltung von okologieorienttierten management instrumenten. Die Unternehmung. (4), 273, 1990.

2. LUO Y.S., LU Z.N., MUHAMMAD S., YANG H. The heterogeneous effects of different technological innovations on eco-efficiency: Evidence from 30 China's provinces. Ecological Indicators. 127, 1, 2021.

3. KHALID K., HUSSAIN B., ALI S. Evaluating ecoefficiency eco-efficiency in consumption and production through sustainable utilization of resources: A panel analysis of APAC by population. Renewable Energy. 170, 1096, 2021. 
4. LIU X.L., GUO P.B., GUO S.F. Assessing the ecoefficiency of a circular economy system in China's coal mining areas: Emergy and data envelopment analysis. Journal of Cleaner Production.. 206, 1101, 2019.

5. GUO Y., LIU W., TIAN J.P., HE R.N., CHE L.J. Ecoefficiency assessment of coal-fired combined heat and power plants in Chinese eco-industrial parks. Journal of Cleaner Production. 168, 963, 2017.

6. LI C.Y., ZHANG S.Q., ZHANG W. Spatial Distribution Characteristics and Influencing Factors of China's Inter Provincial Industrial Eco-efficiency. Scientia Geographica Sinica. 38(12), 1970, 2018.

7. HAN J.P., CHENG X., YAN J., YANG X.L. Study on the Measurement of Urban Eco-industrial Green Development Based on Network Super-efficiency EBM Model: Taking 47 Key Cities in Three Regions and Ten Groups as an Example. Science and Technology Management Research. 39 (05), 228, 2019.

8. ZHANG R.B., REN S.G., CAI L.Y. Industrial Ecoefficiency Evaluation for Urban Agglomeration in the Yangtze River Delta.Commercial Research. (06), 163, 2017.

9. GU P.H., LIU Z.C. Evaluation of Industrial Eco Efficiency Based on Material Flow Analysis - As an Example in Hunan Province. Economic Geography. 37 (04), 141, 2017.

10. LI Y., WANG Q.L., FU L., WANG C.X. Evaluation Research on Economic Efficiency and Ecological Efficiency in Forest Products Industry Ecological Industry Chain: An Empirical Analysis Based on the Three Stage DEA Model in Heilongjiang Province. Ecological Economy. 31 (11), 51, 2015.

11. ZHAO Y., ZHAO C. Discussion on the application of ecological efficiency evaluation to industrial plantation cutting. Forest Engineering. 26 (06), 47, 2010.

12. WANG Y. Measure of Ecological Efficiency and Influencing Factors of the Provinces along the Silk Road Economic Belt - From the Perspectives of FDI and Industrial Structure Optimization. East China Economic Management. 32 (11), 44, 2018.

13. REN X.J., QU X.E. The Regional Ecological Efficiency and the Choice of Environmental Regulation Tools in China - An Empirical Analysis Based on Provincial Panel Data. Journal of Dalian University of Technology(Social Sciences). 41 (01), 28, 2020.

14. YANG G.Q., LI M.Q. Fiscal decentralization, political promotion and energy eco-efficiency improvement: An empirical study of 257 cities in China. Macroeconomics. (08), 41, 2018.

15. TIAN M.Y., HUANG H., ZHANG R.B. An empirical analysis of the impact of industrial transfer on industrial ecological efficiency of urban agglomeration in the middle reaches of Yangtze River. Statistics \& Decision. 34(04), 101, 2018.

16. LIU Z. An Analysis of Eco-efficiency of Regional Green Investment in China Based on Three-stage DEA Model. Economic Survey. 36 (06), 17, 2019.

17. WANG X.L., FANG X.C. Eco-Efficiency Evaluation and Its Influencing Factors for the Old Northeastern Industrial Base of China on DEA-Malmquist-Tobit Model. Ecological Economy. 33 (05), 95, 2017.
18. ZHAO Z., BAI Y.P., HU Z.M., CHEN J.C., DENG X.Z. Evaluation of ecological efficiency and factors influencing grassland animal husbandry in the Hulunbuir region based on a super-efficiency DEA model. Acta Ecologica Sinica. 38 (22), 7968, 2018.

19. MA M., TANG L. Evaluation of Ecological Efficiency of Industrial Chain in Jilin Province under Production Inducement - Based on the Combination of Input-output Method and DEA Method. Taxation and Economy. (01), 103, 2018.

20. HUANG T.H., HU X.Y., CHEN S.F., WANG Y., ZHANG B.Q. Evaluation of the sustainable development level of the Belt and Road countries and its impact factors: based on the Super-SBM and Tobit measure models. China Population, Resources and Environment. 30 (12), 27, 2020.

21. XU W., LI Z.R., ZHANG Y.Y., SHI H.B. An Evaluation of China's Industrial Innovation Efficiency Based on SuperSBM Model and Malmquist Index. Macroeconomics. (05), $55,2021$.

22. WU X.X. Spatial-Temporal Differentiation of Construction Efficiency of Ecological Civilization in Henan Province and Its Origin Tracing. Ecological Economy. 36 (02), 214, 2020.

23. HAN Z.L., WANG Q.W., LIU T.B., YAN X.L. Spatiotemporal Dynamic Distribution and Evolution Trend of China's Ecological Efficiency. Statistics \& Decision. 35 (22), 85, 2019.

24. YANG Y., DENG X.Z. The Spatio-temporal Evolutionary Characteristics and Regional Differences in Affecting Factors Analysis of China's Urban Eco-efficiency. Scientia Geographica Sinica. 39 (07), 1111, 2019.

25. JIANG J., CAO Y.E., ZHAO P.P., ZHANG Z.Z. Study on the spatial and temporal variance of agricultural ecological efficiency in Yili Prefecture, Xinjiang - Based on super efficiency undesirable-SBM model and Malmquist index. Journal of Northwest Normal University (Natural Science). 57 (02), 101, 2021.

26. LUO M., FAN R.G., ZHANG Y.Q. Measurement and Evolution Analysis on China's Environmental and Social Governance Efficiency. Statistics \& Decision. 37 (09), 51, 2021.

27. LI L.Z., LI J.S., JIANG Z.L. Pattern of evolution and formation mechanism analysis of resources and environment efficiency of cities in central china based on SBM-DEA model. Resources and Environment in the Yangtze Basin. 26 (11), 1761, 2017.

28. FANG C.L., REN Y.F. Analysis of emergy-based metabolic efficiency and environmental pressure on the local coupling and telecoupling between urbanization and the eco-environment in the Beijing-Tianjin-Hebei urban agglomeration. Scientia Sinica (Terrae), 47 (07), 833, 2017.

29. WANG K.L., MENG X.R., YANG B.C., CHENG Y.H. The industrial eco-efficiency of the Yangtze River Economic Zone based on environmental pressure. Resources Science. 37 (07), 1491, 2015.

30. LI G., LIU J.G., LI T.Q. Regional Differences of Energy Eco-efficiency in Manufacturing Industry under Consideration of Undesirable Outputs Based on the SBMTobit Two-stage Model. Chinese Journal of Management Science. 27 (11), 76, 2019. 
